\title{
Malpositioning of a Tunneled Hemodialysis Catheter into the Azygos Vein: A Case Report and Literature Review
}

\author{
Li-jun Mou $^{\text {a }}$ Si-hua Wang ${ }^{b}$ Qing-hai Lic Rong-shi Zhang ${ }^{d} \quad$ Nian-ze Pan ${ }^{e}$ \\ Jin-fan Wang ${ }^{e}$
}

${ }^{a}$ Department of Nephrology, ${ }^{b}$ Department of Nursing Education, and ${ }^{\mathrm{c}}$ Department of Radiology, The Second Affiliated Hospital, School of Medicine, Zhejiang University, Hangzhou, and d Department of Hemodialysis Center, and ${ }^{\text {Department }}$ of Internal Medicine, The Second Affiliated Hospital Taijiang Branch, College of Medicine,

Zhejiang University, Taijiang, China

Dear Editor,

The percutaneous catheterization of central veins is increasingly used in nephrological practice as a permanent vascular access. Jugular vein catheterization is considered to have the lowest risk of complications and is usually the site of choice for catheter placement. To achieve the best outcome, the tips of the catheter should be positioned in the superior vena cava, the cavoatrial junction [1]. Inadvertent placement of a hemodialysis catheter into the azygos vein through the right internal jugular vein (RIJV) is rare and difficult to detect [2], and to our knowledge, there were only 3 such cases reported to date [3-5]. We present a case of an uncommon cannulation of the azygos vein through RIJV with a tunneled hemodialysis catheter. A 47-yearold female patient was admitted to the department of Nephrology. She was diagnosed with end-stage renal disease and hemodialysis had to be initiated immediately because nausea and vomiting had developed half a month ago. Therefore, tunneled dialysis catheter was the preferred vascular access for her. The RIJV was easily punc- tured under local anesthesia without any guidance. Nonpulsatile blood flow was obtained before vessel dilation and catheter insertion. A Palindrome $\mathrm{T}^{\mathrm{TM}}$ tunneled catheter $(14.5 \mathrm{Fr}$, symmetrical tip, $36 \mathrm{~cm})$ was placed. The blood from the catheter was aspirated and flushed readily. A routine anteroposterior chest radiograph was performed to assess the catheter tip position following catheter placement. It was noted that the catheter tip kinked apparently, inclined medially at the right tracheobronchial angle (Fig. 1a). Persistent and mild back pain developed. Therefore, malpositioning of the catheter tip into the azygos vein was suspected. An axial CT scan confirmed the suspicion (Fig. 1b).The catheter was withdrawn and placed again through RIJV under the fluoroscopy guidance. The catheter tip was in proper position this time.

Risk factors for malpositioning into the azygos vein include left internal jugular venous access, use of catheters with long venous tips, and catheter insertion in a patient with fluid overload [6]. However, the patient under reference had no fluid over- load; the catheter employed had a symmetrical tip and was placed through RIJV. All risk factors mentioned above failed to explain the occurrence of catheter malpositioning into the azygos vein for the patient discussed in this case report.

Azygos vein cannulation is a rare but hazardous central venous catheter malposition. Perforations in the azygos vein due to inadvertent cannulation were reported in up to $19 \%[6,7]$. In line with this finding, early recognition and prompt correction is crucial. In our patient, ready aspiration of blood from the catheter lumen after its placement did not mean a proper position of the tip [3, 4]. Malpositioning of a hemodialysis catheter into the azygos vein can be difficult to detect during catheter insertion because venograms are not routinely obtained [3]. Guidewire or catheter kinking, or loop-like, buckled appearance at the right tracheobronchial angle is strongly suggestive of malpositioning into the azygos vein [3]. Back pain developed in one previous patient and also in our present patient after the catheter was malpositioned
KARGER

(c) 2017 S. Karger AG, Basel

E-Mail karger@karger.com

www.karger.com/bpu
Rong-shi Zhang

Department of Hemodialysis Center, The Second Affiliated Hospital Taijiang Branch College of Medicine, Zhejiang University, Miaojiangxidadao Road 51

Taijiang, Guizhou Province 556300 (China)

E-Mail mljzju@163.com 

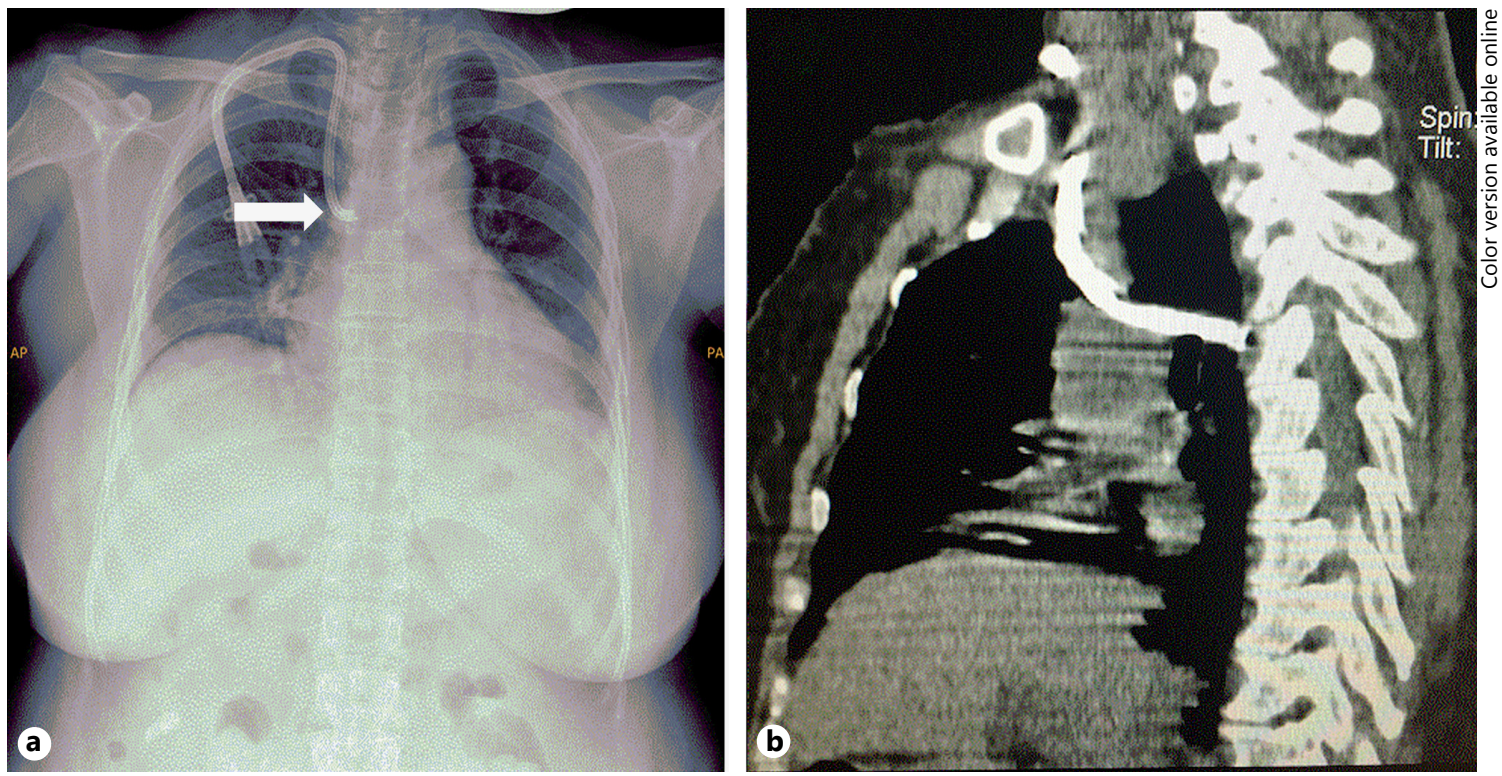

Fig. 1. a A sharp kink (white arrow) and medial deviation in the hemodialysis catheter directly adjacent to the right tracheobronchial angle was noted in the front chest radiograph. b Axial CT scan confirmed the malpositioning of the catheter tip into the azygos vein.

into the azygos vein [8]. Thus, this symptom also indicated the presence of catheter tip malpositioning into the azygos vein.

Malpositioning of a hemodialysis catheter tip into the azygos vein through RIJV is rare. Ready aspiration and flushing of blood from the catheter cannot exclude catheter tip malpositioning. Catheter insertion should be performed under fluoroscopy control or at least controlled by chest X-ray after insertion and before use to check the right position of the catheter tip. When catheter kink occurs at the right tracheobronchial angle and the patient complains of back pain, a catheter tip malpositioning into azygos vein should be suspected.

\section{Ethics}

The patient had given her informed consent to be part of this study and this study protocol was approved by our hospital's committee on human research.

\section{Acknowledgments}

This work was supported by research grants from the Public Technology Application Research of Zhejiang Province (2017C33093).

\section{Disclosure Statement}

The authors have declared no conflicts of interest.

\section{References}

1 Vascular Access Work Group. Clinical practice guidelines for vascular access. Am J Kidney Dis 2006;48(suppl 1):S248-S273.

2 Marcy PY, Guiffant G, Merckx J, Lacout A: Diagnosis of azygos catheter misplacement. J Vasc Access 2016;17:e148-e149.

3 Pua U: Imaging teaching case. Radiographic features of malpositioning of a hemodialysis catheter in the azygos vein. Am J Kidney Dis 2010;55:395-398.

4 Calvino J, Bravo J, Martinez L, Millan B, Pulpeiro JR: Recognizing misplacement of a dialysis catheter in the azygos vein. Hemodial Int 2013;17:455-457.

5 Nayeemuddin M, Pherwani AD, Asquith JR: Imaging and management of complications of central venous catheters. Clin Radiol 2013; 68:529-544.

6 Bankier AA, Mallek R, Wiesmayr MN, et al: Azygos arch cannulation by central venous catheters: radiographic detection of malposition and subsequent complications. J Thorac Imaging 1997;12:64-69.

7 Harish K, Madhu YC: Inadvertent port: catheter placement in azygos vein. Int J Angiol 2012;21:103-106.

8 Rosa UW, Foreman M, Willsie-Ediger S. Intermittent back pain after central venous catheter placement. JPEN J Parenter Enteral Nutr 1993;17:91-93. 